BNL-114214-2017-JA

\title{
Optical Asymmetry and Nonlinear Light Scattering from Colloidal Gold Nanorods
}

\author{
M.-B. Lien, M.-G. Han
}

Submitted to ACS NANO

June 2017

\author{
Condensed Matter Physics and Materials Science Department \\ Brookhaven National Laboratory
}

\author{
U.S. Department of Energy \\ USDOE Office of Science (SC), \\ Basic Energy Sciences (BES) (SC-22)
}

Notice: This manuscript has been authored by employees of Brookhaven Science Associates, LLC under Contract No. DE- SC0012704 with the U.S. Department of Energy. The publisher by accepting the manuscript for publication acknowledges that the United States Government retains a non-exclusive, paid-up, irrevocable, world-wide license to publish or reproduce the published form of this manuscript, or allow others to do so, for United States Government purposes. 


\section{DISCLAIMER}

This report was prepared as an account of work sponsored by an agency of the United States Government. Neither the United States Government nor any agency thereof, nor any of their employees, nor any of their contractors, subcontractors, or their employees, makes any warranty, express or implied, or assumes any legal liability or responsibility for the accuracy, completeness, or any third party's use or the results of such use of any information, apparatus, product, or process disclosed, or represents that its use would not infringe privately owned rights. Reference herein to any specific commercial product, process, or service by trade name, trademark, manufacturer, or otherwise, does not necessarily constitute or imply its endorsement, recommendation, or favoring by the United States Government or any agency thereof or its contractors or subcontractors. The views and opinions of authors expressed herein do not necessarily state or reflect those of the United States Government or any agency thereof. 


\section{Optical Asymmetry and Nonlinear Light Scattering from Gold Nanorods}

Miao-Bin Lien ${ }^{a}$, Ji-Young Kim ${ }^{b}$, Myung-Geun Han ${ }^{c}$, You-Chia Chang ${ }^{d}$, Yu-Chung Chang ${ }^{e}$,

Heather J. Ferguson ${ }^{a}$, Yimei Zhu ${ }^{c}$, John C. Schotland ${ }^{f}$,Nicholas A. Kotov ${ }^{b}$, Theodore B. Norris ${ }^{a, *}$

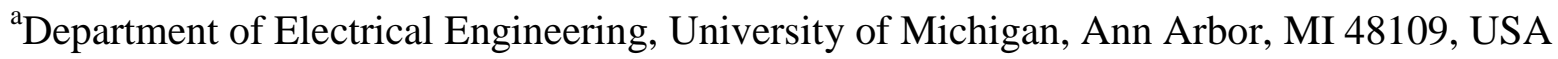

${ }^{\mathrm{b}}$ Department of Materials Science and Engineering, University of Michigan, Ann Arbor, MI 48109, USA

${ }^{c}$ Condensed Matter Physics and Materials Sciences Department, Brookhaven National

Laboratory, Upton, NY 11973, USA

${ }^{\mathrm{d}}$ Department of Physics, University of Michigan, Ann Arbor, MI 48109, USA

${ }^{\mathrm{e}}$ Department of Electrical Engineering, National Changhua University of Education, Taiwan

${ }^{\mathrm{f}}$ Department of Mathematics, University of Michigan, Ann Arbor, MI 48109

*Correspondence: tnorris@umich.edu 


\section{ABSTRACT:}

A systematic study is presented of the intensity-dependent nonlinear light scattering spectra of gold nanorods under resonant excitation of the longitudinal surface plasmon resonance (SPR). The spectra exhibit features due to coherent second and third harmonic generation as well as a broadband feature that has been previously attributed to multiphoton photoluminescence arising primarily from interband optical transitions in the gold. A detailed study of the spectral dependence of the scaling of the scattered light with excitation intensity shows unexpected scaling behavior of the coherent signals, which is quantitatively accounted for by optically induced damping of the SPR mode through a Fermi liquid model of the electronic scattering. The broadband feature is shown to arise not from luminescence, but from scattering of the secondorder longitudinal SPR mode with the electron gas, where efficient excitation of the $2^{\text {nd }}$ order mode arises from an optical asymmetry of the nanorod. The electronic-temperature-dependent plasmon damping and the Fermi-Dirac distribution together determine the intensity dependence

of the broadband emission, and the structure-dependent absorption spectrum determines the spectral shape through the fluctuation-dissipation theorem. Hence a complete self-consistent picture of both coherent and incoherent light scattering is obtained with a single set of physical parameters.

\section{KEYWORDS:}

Plasmonics, Nonlinear Optics, Nanophotonics, Condensed Matter Science 
Nanoscale noble metal structures, such as gold and silver nanoparticles, exhibit optical and electronic properties that are not observed in their bulk counterparts. The electromagnetic modes of these systems, known as surface plasmon resonances (SPRs), lead to strong interaction with light, and an enhancement of the optical field enabling nonlinear effects to be observed with low incident light intensity ${ }^{1,2}$. The plasmon resonances and the field enhancement can be tailored through the choice of metal, the specific size and shape, the dielectric environment, and their mutual spatial arrangement ${ }^{3-5}$. In fact, the sensitivity of the resonances to the local dielectric and charge environment enables the SPR to serve as an optical interrogation of the environment and thus as an optical sensor. The attachment of chemical ligands enables selective sensing of chemical or biomedical targets. As a result, metal nanoparticles serve as important building blocks in the fields of modern chemical engineering ${ }^{6}$, biosensors ${ }^{7}$ and advanced microscopy ${ }^{8-}$ 10. A fundamental understanding of the nonlinear optical response of metal nanostructures is thus essential for the development of optical applications of plasmonics.

The two principal nonlinear optical responses that can be observed on direct far-field excitation are multi-photon luminescence (PL) and harmonic generation (HG). Both phenomena have been investigated extensively. Early observations of two-photon luminescence (TPL) were reported by Boyd et al ${ }^{11}$, and interpreted as the generation and recombination of electron-hole pairs involving the d-band and the conduction (sp) band. The luminescence efficiency of gold nanoparticles can be remarkably high ${ }^{12}$, suggesting applications to biological labeling and imaging ${ }^{13}$. More recently, harmonic generation from metal nanoparticles has received considerable attention. Lippitz et al. reported the first observation of third-harmonic generation (THG) from individual gold colloidal particles down to $40 \mathrm{~nm}$ diameter ${ }^{14}$, and third harmonic 
imaging experiments with gold nanorods was demonstrated in refs ${ }^{14,15}$. A phenomenological theory of second harmonic generation (SHG) from centrosymmetric spherical metallic particles was developed by J. I. Dadap et al ${ }^{16,17}$. Starting with the second order nonlinear surface susceptibilities, they presented a general electro-magnetic theory of SH Rayleigh scattering (SHRS) for spheres within the small particle limit, including the contribution from the bulk nonlinear response compatible with isotropic symmetry. They predicted a quadrupolar-type radiation pattern, as the second order process is in principle dipole-forbidden in centrosymmetric material with centrosymmetric geometry, consistent with experimental results on hyper-Rayleigh scattering from silver nanospheres reported by Hao et al ${ }^{18}$ and a series of polarization analysis experiments performed by Brevet et al ${ }^{19-21}$.

\section{Results and Discussion}

On optical excitation with ultrashort optical pulses, gold nanorods (AuNRs) exhibit hyperRayleigh scattering (SHG and THG) as well as a broadband feature attributed to PL. An example of the scattered light spectrum under 800-nm 50-fs excitation in our experiments (discussed in detail below) displays these features as shown in Figure 1(a). These responses have been respectively interpreted in terms of conventional nonlinear optical processes ${ }^{14,22}$ and multiphoton interband luminescence processes ${ }^{12,23-25}$. There are, however, features of the data whose interpretation is problematic with these physical models. The SHG signal, for example, is surprisingly strong, whereas in contrast to the prediction that, in the small particle limit, SHG should be forbidden given the assumed centrosymmetry of the system; the observed SHG has rather been attributed to the effects of either the metal-media interface or retardation ${ }^{16-21}$. Additionally, the SHG and THG signals from AuNRs show saturation-like characteristics in the 
power scaling (see Fig 1b and 1c), which has been ambiguously attributed to damage of the nanoparticles ${ }^{15}$. The broadband spectral feature that has been attributed to PL in gold nanoantennas has recently been recently shown to depend primarily on the nanoscale geometry, and does not in fact arise from the electronic band structure ${ }^{26}$. Finally, the nonlinear response has been theoretically predicted to exhibit unusual scaling with excitation intensity due to surface confinement effects on the electrons undergoing collective oscillation in the SPR ${ }^{27}$.

Among the most common forms of structure used for studies of nanoplasmonics are chemically synthesized AuNRs, grown in aqueous solution guided by the surfactant cetyltrimethylammonium bromide (CTAB) ${ }^{28}$. A spatial analysis of the electrostatic potential around these AuNRs was recently performed using off-axis electron holography ${ }^{29}$. In the absence of magnetic field, the phase shift $\phi(x, y)$ in an electron wave after passing through a specimen is proportional to a line integral of the electrostatic potential along the propagation direction ${ }^{30}$. The reconstructed phase shift $\phi(x, y)$ map obtained for a AuNR shows a considerable asymmetry and a distinctive slope along the long axis of the rod (see Fig. 2a and 2b), indicating a presence of static dipole moment. In our recent study ${ }^{31}$, we have found that non-uniform coverage of the surfactant CTAB induces a static dipole moment, which breaks the inversion symmetry and leads to an optical asymmetry. This has far-reaching consequences, since for an optically symmetric nanoparticle, SHG is forbidden to lowest order, and can arise only through the effect of retardation and quadrupole radiation. We show in this paper that the optical symmetry breaking by the static dipole in AuNRs enables a novel, quantitative, and selfconsistent explanation of all the major features of nonlinear light scattering. 
Motivated by these considerations, we have performed a systematic series of nonlinear light scattering experiments, with the goal of obtaining a physical model that can simultaneously, selfconsistently, and quantitatively explain all the main features of the SHG, THG, and broadband signals observed in Figure 1.

The experimental setup is shown in Figure 3. The excitation source is a Ti:sapphire modelocked laser, which provides 800 -nm, 50-fs pulses at a repetition rate of $76 \mathrm{MHz}$. The excitation power is controlled by a variable attenuator consisting of a half-wave plate mounted on a programmable rotator and a Glan-Thompson polarizer. The laser pulses pass through a prism compressor so that the pulses are transform-limited at the focal point. A small fraction of the beam is sampled by a photodiode in order to measure the incident power and its fluctuations. In addition, a $780 \mathrm{~nm}$ high-pass filter is placed before the focusing lens to ensure there is no residual SHG or THG in the excitation beam. The beam is focused in the center of a cuvette containing an aqueous solution of AuNRs with a longitudinal plasmon resonance at $808 \mathrm{~nm}$. Light scattered at $90^{\circ}$ from the excitation direction is collected by an $\mathrm{f}^{\prime \prime}=1$ uv-coated lens and filtered through either BG filters (BG-39, BG-40 color filters) or $267 \mathrm{~nm}$ band-pass filters to remove the linear $800 \mathrm{~nm}$ scattering. The collected signal is focused on the entrance slit of a monochromator and detected by a single photon counting photomultiplier. A chopper is placed in the excitation beam path to modulate the excitation beam in order to subtract background dark counts from the signal in gated photon counting mode.

A typical spectrum of the nonlinearly scattered light is shown in Figure 1(a), showing clearly three main components: THG and SHG peaks (hyper-Rayleigh scattering) located at $267 \mathrm{~nm}$ and 
$400 \mathrm{~nm}$, respectively, and a broadband feature ranging from $350 \mathrm{~nm}$ to $600 \mathrm{~nm}$ that is generally referred to as PL. We note that the SHG peak overlaps with the broadband photoluminescence. A key experiment to revealing the physical processes underlying the light scattering is the scaling of the various spectral components with excitation intensity (or fluence in the case of fixed pulse duration). Figure 1 (b) and 1 (c) shows the measured scaling of the SHG and THG peaks. On the basis of the standard perturbation theory of nonlinear optics ${ }^{32}$, the SHG should scale exactly as the square of the intensity, and the THG as the cube.

The THG signal has a well-defined spectral peak at $267 \mathrm{~nm}$ and is background-free (Figure 1 inset). A fit to a scaling curve of the form $I_{\text {sig }}=a\left(I_{e x}\right)^{b}$ yields an exponent of $2.46( \pm 0.022)$, quite different from the expected value of 3 . The SHG peak at $400 \mathrm{~nm}$ sits on top of the broad PL background. To determine the SHG scaling, we subtract the PL before integrating the area under the 400-nm peak (the assumption that the PL spectrum is smooth is fully justified by the model below). The background-subtracted SHG scales with an exponent of $1.49 \pm 0.03$, far from its conventional expected value of 2.

The unexpected scaling of the SHG and THG signals can be explained quantitatively by optically induced damping of the longitudinal SPR by hot electrons. ${ }^{33,34}$. In a model of the SPR mode of the AuNR as a classical anharmonic oscillator, the second and third order nonlinear susceptibilities $\chi_{2}$ and $\chi_{3}$ are related to the linear susceptibility $\chi_{1}$ by

$$
\begin{aligned}
& \chi^{(2)}(\omega) \propto \chi^{(1)}(2 \omega)\left[\chi^{(1)}(\omega)\right]^{2} \text { and } \chi^{(3)}(\omega) \propto \chi^{(1)}(3 \omega)\left[\chi^{(1)}(\omega)\right]^{3} \text { where } \\
& \chi^{(1)}(\omega)=\frac{N\left(e^{2} / m\right)}{\epsilon_{0} D(\omega)}, D(\omega)=\omega_{0}^{2}-\omega^{2}-2 i \omega \gamma \text { and } \omega_{0} \text { is the material resonance frequency }
\end{aligned}
$$


The factor $D(\omega)$ contains the resonance frequency of the system (corresponding to $808 \mathrm{~nm}$ wavelength for the longitudinal plasmon mode of the AuNR) and the damping rate $\gamma$. It has been shown that scattering of the nanoparticle plasmon from single-particle excitations of the electron gas is the dominant nonlinearity in the optical response, as $\gamma$ depends on the electron temperature ${ }^{34}$. Following the Fermi liquid model of ref ${ }^{34}$, we find the intensity scaling will be modified by the pump-induced electron heating according to

$$
\begin{aligned}
& I_{S H G} \propto \chi^{(2)}(\omega) I^{2} \propto\left[I / \gamma_{p}\right]^{2} \text { and } I_{T H G} \propto \chi^{(3)}(\omega) I^{3} \propto\left[I / \gamma_{p}\right]^{3} \text { where } \\
& \gamma_{p} \propto\left\langle\gamma_{e e}\right\rangle=\frac{\int_{0}^{\infty} f(\varepsilon) \gamma_{e e}(\varepsilon) d \varepsilon}{\int_{0}^{\infty} f(\varepsilon) d \varepsilon} \text { is the total electron-temperature-dependent scattering rate and } \\
& \gamma_{e e}\left(\varepsilon, T_{e}\right)=\gamma_{0}+\frac{K\left(\pi k_{B} T_{e}\right)^{2}+\left(\varepsilon-\varepsilon_{F}\right)^{2}}{1+\exp \left[-\left(\varepsilon-\varepsilon_{F}\right) / k_{B} T_{e}\right]}
\end{aligned}
$$

The model has only two fitting parameters, namely $\gamma_{0}$, the electronic-temperature-independent part of $\gamma_{e e}$, and $U_{a b s}$, the absorbed pulse energy per AuNR that results in heating the conduction band electrons ${ }^{35}$. The SHG and THG scaling fit the model very well (data fits are 1.49, 2.46 and the model predictions are 1.47, 2.41 for SHG and THG respectively) for a single value of $\gamma_{0}=0.067\left(\mathrm{fs}^{-1}\right)$ and $U_{a b s} \approx 0.267 U_{t h}$, where $U_{t h}$ is the absorbed energy estimated from the estimated incident intensity and a classical model of the absorption cross section of a AuNR ${ }^{4}$. In fact, it can be seen in Figure 1 (c) and 1(d) that the Fermi liquid model fits the scaling data better than an assumed power law scaling. We conclude that the SPR damping induced by a hot thermal distribution of single-particle excitations quantitatively accounts for the unexpected scaling of the nonlinear optical response of the AuNR longitudinal plasmon resonance. 
We now discuss the broadband scattered light, which has generally been attributed to PL arising from the recombination of d-band holes with conduction band electrons excited by multiphoton absorption in prior work. Our key new experiment is the measurement of the complete spectral dependence of the intensity scaling of the broadband emission, as shown in Figure 4(b). The scattered light scales with an exponent of approximately 3.6 at $375 \mathrm{~nm}$ to 2.5 above $550 \mathrm{~nm}$; i.e. the shorter wavelength emission exhibits a more highly nonlinear behavior. Interestingly, the total signal at $400 \mathrm{~nm}$ (i.e. the sum of the coherent SHG and the broadband signal) does not follow the general trend, but scales with an exponent of 1.92. While multiphoton PL from interband transitions can account for some features of the spectrum ${ }^{1,8,12,36}$, others are inconsistent with PL as the source of the broadband emission. In particular, the spectral features of the broadband spectrum from AuNRs do not really match well with those from their bulk counterpart, though some previous work has attempted to attribute these features to recombination processes between conduction band electrons and d-band holes near the $\mathrm{X}$ and $\mathrm{L}$ symmetry points in the Brillouin zone ${ }^{8,23-25}$. Recent experiments comparing PL from gold nanoantennas fabricated from single crystals and polycrystalline films, however, have suggested that it is the nanoscale geometry of the plasmonic structures that determines the shape of the emission spectra ${ }^{26}$, and not the band structure. Most importantly in this study, we performed an additional set of experiments in which the AuNRs were excited directly with 400-nm 50-fs pulses (generated by frequency doubling the Ti:sapphire laser), which should induce one-photon absorption energetically equivalent to 2-photon absorption at $800 \mathrm{~nm}$. The threshold for absorption from the $\mathrm{d}$ bands in $\mathrm{Au}$ is $1.8 \mathrm{eV}$; hence $800-\mathrm{nm}$ excitation from the d-band requires two- or three-photon absorption, while linear absorption of 400-nm light can excite d-band holes. 
We found there was only negligible emission from the AuNRs under 400-nm excitation even at the highest accessible excitation intensity; this indicates unequivocally that the broadband emission under 800-nm excitation does not arise from interband transitions as is usually thought.

Given that the broadband emission is not in fact interband PL, we propose a new model in which the nonlinear light scattering arises from an interplay of the plasmon damping and the optical anisotropy due to the presence of a static dipole on the AuNRs. On illumination with 800$\mathrm{nm}$ pulses, the gold nanorod's longitudinal plasmon mode, corresponding to a collective oscillation of the electrons along the long axis of the particle, is resonantly excited. The charge asymmetry associated with the static dipole along the nanorod axis gives rise to a nonzero $\chi^{(2)}$; the second-order nonlinear polarization along the nanorod axis excites the asymmetric secondorder longitudinal plasmon mode, which can efficiently scatter with the background free electron gas just as the fundamental plasmon mode scatters, as described above.

The process is illustrated in Figure 5(a), indicating how two longitudinal plasmons excited by the pump give rise to a fluctuating polarization $P_{\omega}$ due to scattering from the free electron gas. Electrons oscillating in opposite directions in the asymmetric second-order longitudinal mode scatter with free carriers, producing dipole fluctuations along the transverse nanorod axis, see Figure 5(a) inset. The emission arises as radiation from $P_{\omega}$, and the coupling of these dipole fluctuations to free-space electromagnetic modes is enhanced by the transverse plasmon mode of the AuNRs. As illustrated in Figure 5(a), a continuum of radiation is produced since the scattering of an electron with initial energy $\varepsilon-\Delta / 2$ to a final state with energy $\varepsilon+\Delta / 2$ results in a shift $\Delta$ of the emission relative to the second harmonic of the $800-\mathrm{nm}$ excitation; the 
broadband spectrum arises from an integral over all possible initial and final free-carrier states with occupations $f_{i}$ and $f_{f}$ determined by the Fermi function for a carrier gas with electron temperature $T_{e}$, which is well-known from the scaling experiments on SHG and THG discussed above. Since the nonlinear light scattering is driven by scattering of plasmons from free carriers, the total emission should be proportional to the plasmonic damping rate, $\gamma_{p} \propto\left\langle\gamma_{e e}\right\rangle$. Finally, the scattered spectrum $I(\omega)$ is proportional to a lineshape factor $L(\omega)$ given by the Fourier transform of the dipole-dipole correlation function, and, by the fluctuation-dissipation theorem, this is proportional to the imaginary part of the susceptibility, i.e. the absorption spectrum ${ }^{37,38}$; in the calculation we use the actual experimental absorption spectrum of the AuNR sample. Putting all these elements together, the broadband scattered light will be given by the expression

$$
\begin{aligned}
& I(\lambda, U)=I\left(\omega, T_{e}\right) \propto \int f(\varepsilon-\Delta / 2)[1-f(\varepsilon+\Delta / 2)] d \varepsilon \cdot \gamma_{e e}\left(T_{e}\right) \cdot L(\omega) \\
& \text { where } \Delta=2 \hbar \omega_{800 \mathrm{~nm}}-\hbar \omega \text { and } f(\varepsilon)=\frac{1}{1+\exp \frac{\left(\varepsilon-\varepsilon_{F}\right)}{k_{B} T_{e}}}
\end{aligned}
$$

is the Fermi function with electron temperature $T_{e}(U)$.

Figure 5(b) shows the calculated scattered light spectrum using the values of $\gamma_{e e}$ and $U_{a b s}$ obtained from the SHG and THG experiments; Figure 1(a) shows the close match of the theory (red line) and experimental (blue dots) line shapes. Note that the spectral smoothness around the SHG position justifies the interpolation and subtraction of the background from the SHG peak around $400 \mathrm{~nm}$ discussed above. Most critically, we can also generate from the calculation the intensity scaling as a function of wavelength; the result shown as the red curve in Figure 4(b), and shows excellent agreement with the data. This is a strong confirmation of the model, indicating that interband PL processes do not play a significant role in the multiphoton 
broadband emission, but that second-order nonlinear light scattering mediated by damping of the second-order longitudinal plasmon resonance is mainly responsible for the scattered light. It should be noted the only fitting parameter in the calculation is the value of the Fermi level; the fitting leads to $\varepsilon_{F}=5.4(\mathrm{eV})$, which is very close to the bulk value of $5.5(\mathrm{eV})$, and the slight difference may be due to pump-induced heating. A final confirmation that the second-order light scattering mechanism described above is correct is the observation that excitation at $400 \mathrm{~nm}$ only leads to negligible broadband emission; excitation at $400 \mathrm{~nm}$ does not induce an excitation of the second order plasmon mode which could then scatter into a fluctuating dipole transverse to the nanorod.

\section{Conclusion}

In summary, we have systematically investigated nonlinear light scattering from AuNRs under resonant excitation of the longitudinal plasmon mode, with detailed measurements of the spectral dependence of the intensity scaling across the SHG, THG, and broadband components. From our recent electron holography study (reported elsewhere), we know that the structurally symmetric gold nanorods are in fact electrically and optically anisotropic due to asymmetric charge distributions induced by the ligands used to guide their self-assembly. This optical asymmetry leads to strong second-order polarizability $\chi^{(2)}$ and hence the significant SHG signal. The SHG and THG nonlinear susceptibilities are intensity dependent, through the optically induced damping of the SPR linear susceptibility, corresponding to scattering of the plasmon with hot electrons; this damping is quantitatively understood through the electron temperature dependence in the Fermi liquid model. Because of the nonzero $\chi^{(2)}$, the second-order longitudinal mode is excited. Scattering of this mode from the electron gas results in a fluctuating polarization along 
the transverse direction of the nanorod, which radiates a broadband spectrum. The spectrum as well as the intensity scaling are understood through a self-consistent model of light scattering. Interband transition processes apparently play no significant role in the light emission. We note finally that the model incorporates the actual SPR spectrum through the fluctuation-dissipation theorem and assumes scattering from a perfectly thermalized electron gas, and hence does not account for the individual microscopic scattering processes responsible for the radiation. It thus cannot account for the possible role of coherence in the scattering process, which may be important for the complete interpretation of the emission around $400 \mathrm{~nm}$; the nearly quadratic scaling of the total signal at $400 \mathrm{~nm}$ (SHG + broadband component) is highly suggestive, and remains the subject for additional study.

\section{METHODS:}

\section{GNR sample and the damage testing}

The data presented in this paper are from samples consisting of aqueous solutions of AuNRs purchased from Nanopartz. The solution concentration is approximately $1 \mathrm{nM}$, sufficiently low that particle interaction effects are neglible. The AuNRs have diameter 25 nm and length 102 $\mathrm{nm}$, leading to a transverse plasmon resonance at $517 \mathrm{~nm}$ and longitudinal plasmon resonance at $808 \mathrm{~nm}$. The solution sample is placed in a UV transparent cuvette during the optical excitation to avoid the absorption of the scattered nonlinear optical signal.

We rule out the possibility of rod melting by monitoring the difference between the initial and final UV-VIS spectra of the gold nanorod solution sample. Gold nanorods in solution can be melted into spherical nanoparticles by excitation with intense femtosecond laser pulses of 
sufficient energy. If the energy absorbed by the rods are strong enough such that the rods are partially melted before diffusing out of the focal volume, the longitudinal absorption band would blue-shift, as the aspect ratio of the rods would decrease. If the absorbed energy is even larger such that the rods completely melt into spheres before diffusing out of the focal volume, the intensity of the longitudinal absorption band would decrease, while the intensity of the transverse absorption band would increase, suggesting the depletion of the gold nanorods and the formation of spherical nanoparticles. This phenomenon has previously been observed and reported as a valid method to monitor the rod shape change ${ }^{39}$. Indeed, even with the most intense excitation in our experiment, no blue-shift or the decrement of the longitudinal absorption intensity in the final UV-VIS spectrum was observed, indicating that no rod melting occurred.

\section{Intensity dependence measurement}

The measurement of the intensity scaling corresponds to the determination of $\gamma$ from $y=a x^{\gamma}$ where $y$ represents the optical signal and $x$ represents the excitation intensity. The task is essentially the extraction of the fitted linear slope from the data points in a log-log plot, which must be performed in such a way as to obtain a well-defined error in the determined scaling coefficient. Consider several data points measured with increasing incident intensities. The integration time at each incident intensity is varied to control the error bar on each of the data points, such that the error bars on the log-log plot have equal width. This can be achieved by making the signal-noise-ratio (SNR) the same at every data point via the number of measurements at each data point. Let $\mu$ be the population mean, $\bar{x}$ be the sample mean, $\sigma^{2}$ be

population variance and $s^{2}$ be sample variance. With $n$ samples, we have $\mu=\bar{x} \pm t_{n-1} \frac{s}{\sqrt{n}}=\bar{x} \pm$ $\Delta x$ for $95 \%$ confidence under t-distribution, and we define $S N R=K=\bar{x} / \Delta x$. Now $\bar{x}$ and $s$ can 
be obtained through measurements and the estimated number of samplings $n$ to reach the desired $S N R=K$ is $n=\left[\frac{K \cdot t_{n-1} s}{\bar{x}}\right]^{2}$. With these definitions, we developed the following procedure to reach a desired $S N R=K$ :

1. Take $n_{0}=10$ measurements and calculate $\bar{x}_{10}$ and $s_{10}$. In our case we collect photon counts in 5 sec segments for 10 times.

2. Compare $K_{10}=\bar{x}_{10} / t_{10-1} \frac{s_{10}}{\sqrt{10}}$ to the desired $S N R, K$, where $t_{9}=2.262$. If $K_{10}>K$, we conclude that the desired $S N R$ is reached and take $\bar{x}_{10}$ as the signal count rate.

3. If $K_{10}<K$, we enlarge $n_{1}$ by $n_{1}=\left[\frac{K \cdot t_{10-1} s_{10}}{\bar{x}_{10}}\right]^{2}$

4. Take additional $n_{1}-n_{0}$ points. Calculate $\bar{x}_{n_{1}}, s_{n_{1}}$ and $K_{n_{1}}$. Note $\bar{x}_{n}$ and $s_{n}$ will converge as $n \rightarrow \infty$.

5. Repeat $2-4$.

The procedure ensures that on each data point, the error bars on the log-log plot are with equal width $\log \frac{K+1}{K-1}$, hence minimizing the variance of the extracted fitted linear slope on the log-log plot.

\section{FIGURES:}


(a)

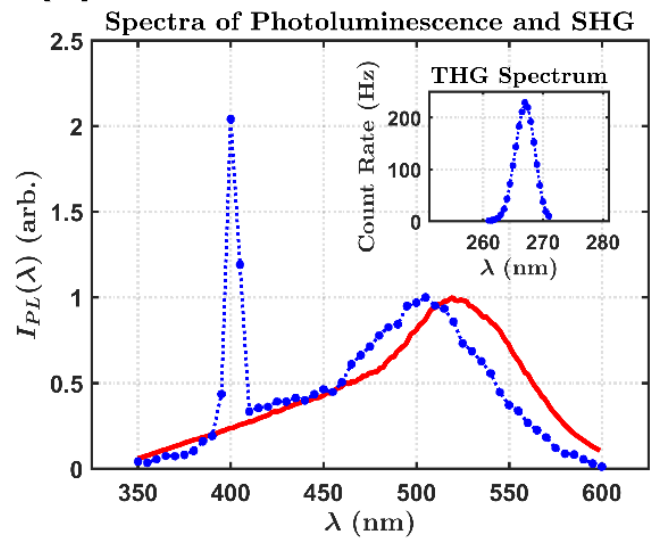

(b)

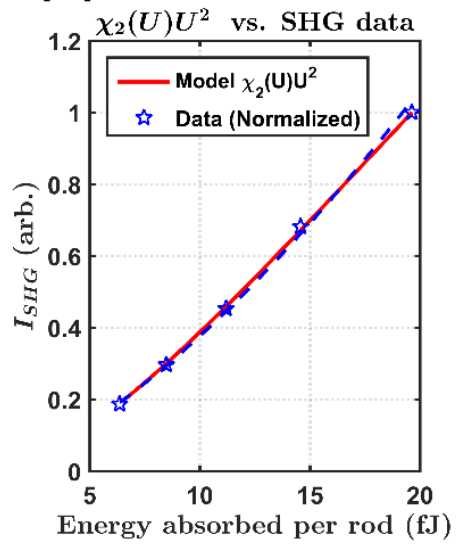

(c)

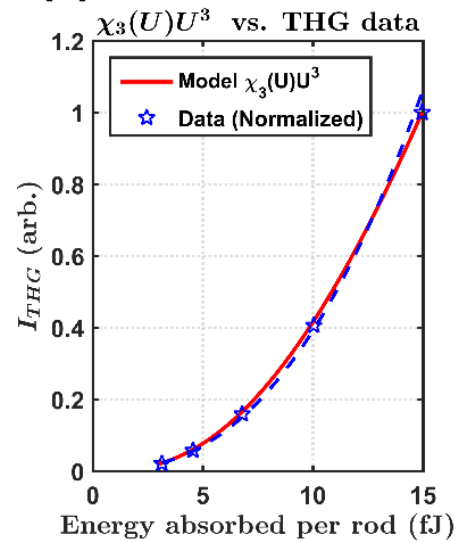

Figure 1. Nonlinear optical response on resonant excitation with 800 -nm, 50-fs ultrashort pulses (a) the spectral components include SHG, THG (inset) and a broadband PL. The red curve is the calculated spectrum with the proposed model (b) the unconventional intensity scaling of SHG (with the background PL subtracted) and (c) THG (background free). The blue dashed lines are the exponential fittings of the data and the red curves are the calculated SHG and THG with optically induced damping of the longitudinal SPR model. Notice the red curves fit the data better than the exponential fitting. 
(a)

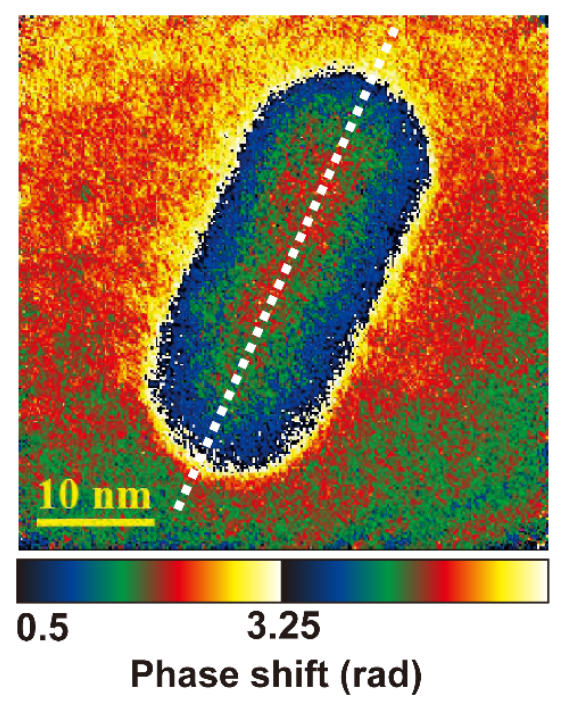

(b)

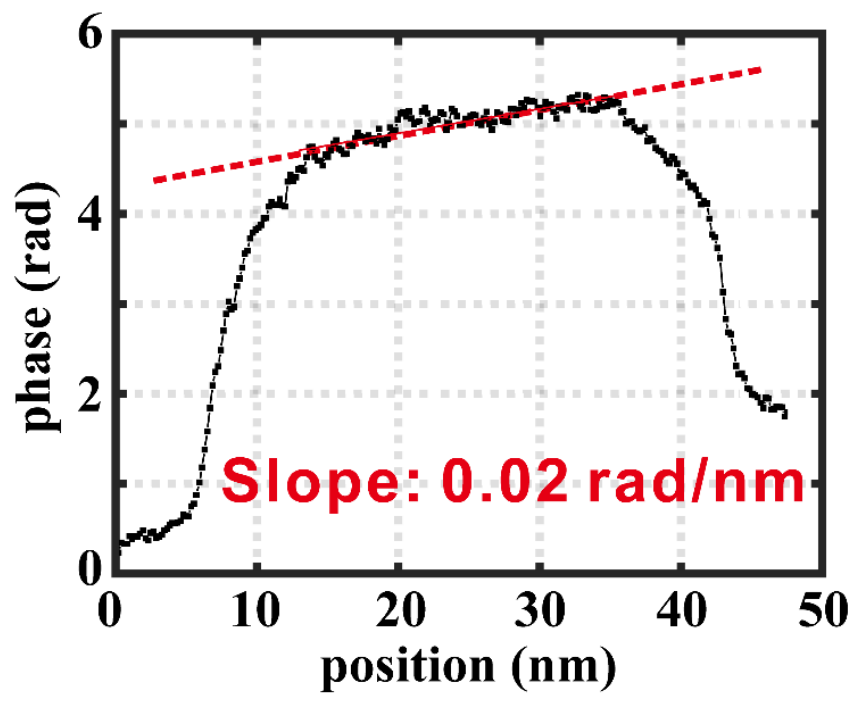

Figure 2. (a) False-color reconstructed phase shift image and (b) its line profile along the long axis (white dashed line in a.) of the AuNR.

Compressor Set

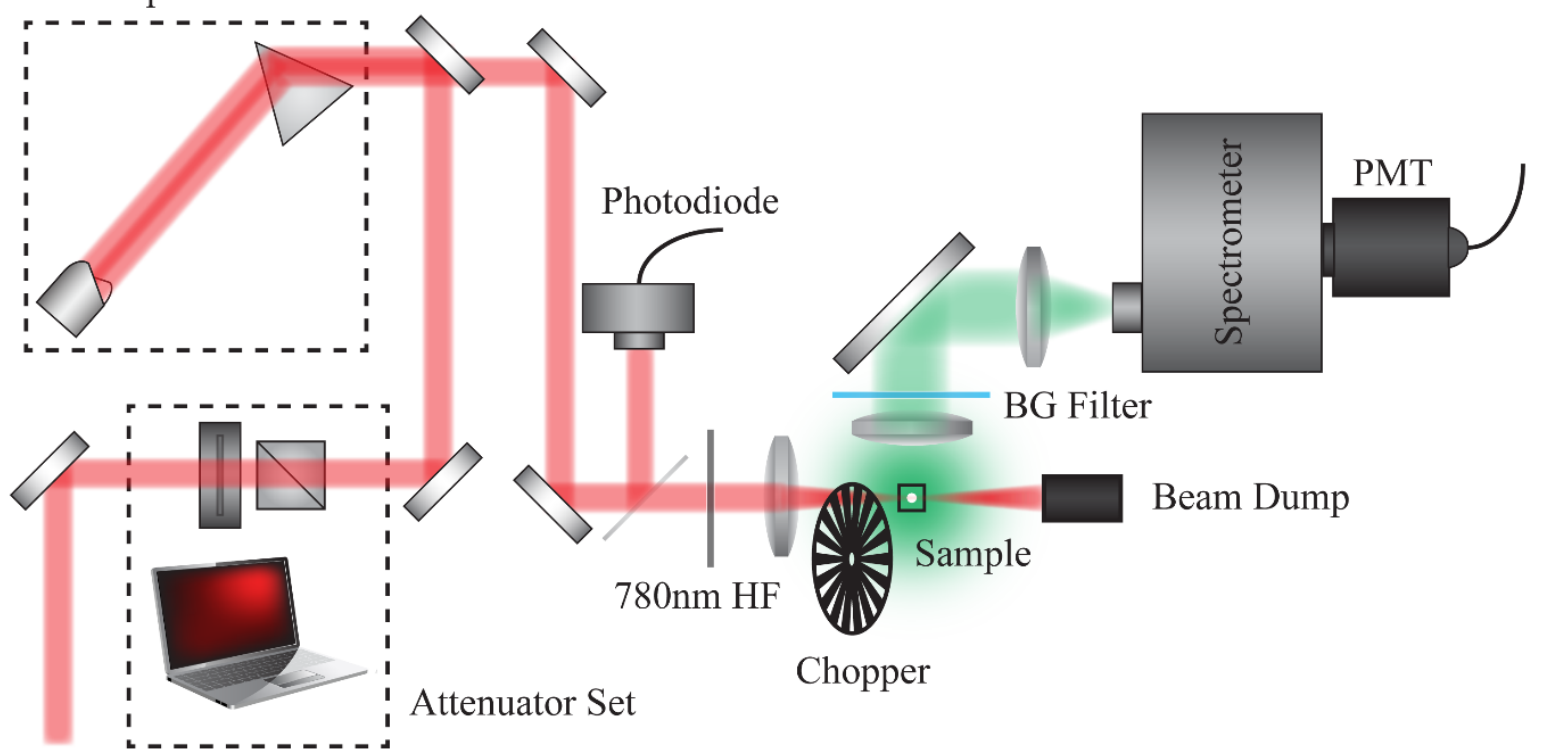

Figure 3. The experimental setup for the spectral and intensity dependence study on the nonlinear light scattering from AuNRs. 
(a)

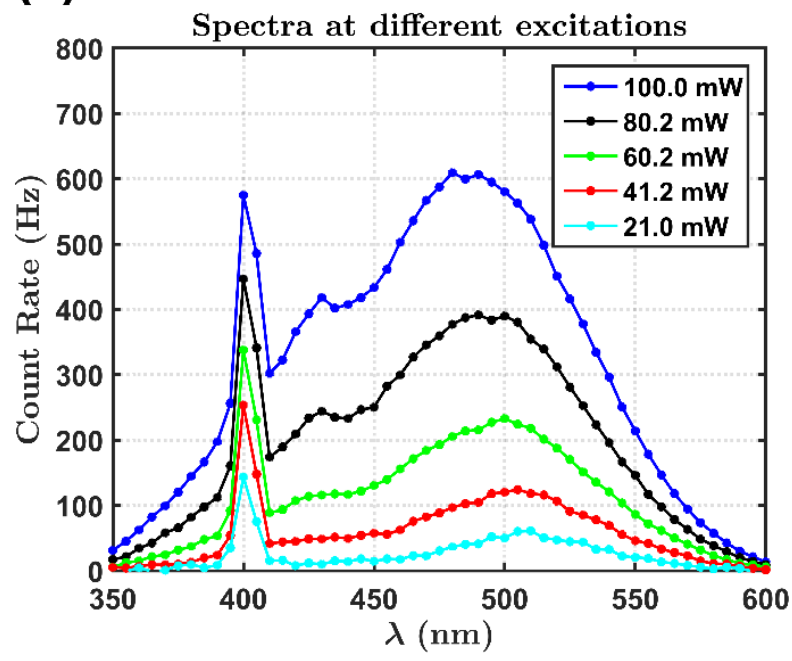

(b)

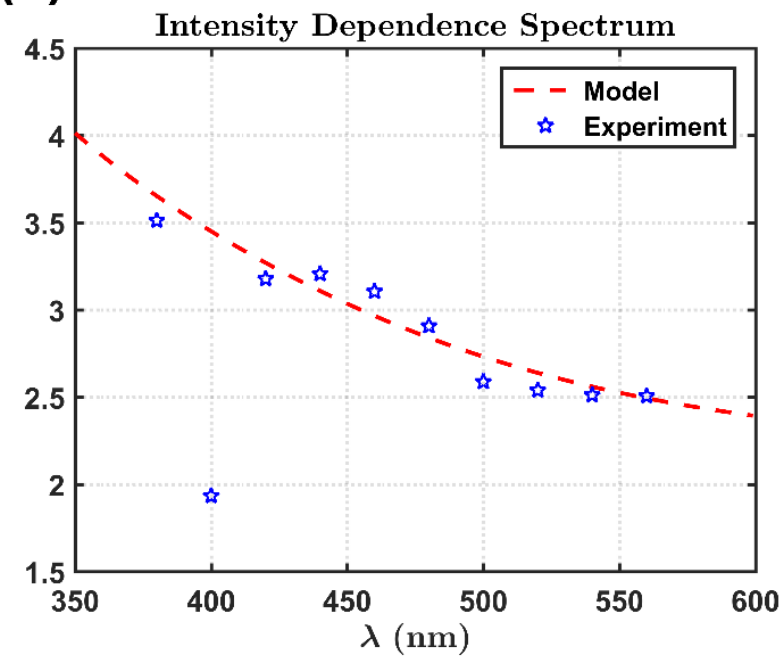

Figure 4. (a) The spectra of nonlinear optical responses measured at different excitation intensities (b) The spectrally resolved intensity dependent scaling. Notice the scaling at $400 \mathrm{~nm}$ is close to 2. The red dash curve is generated from the proposed model.

(a)

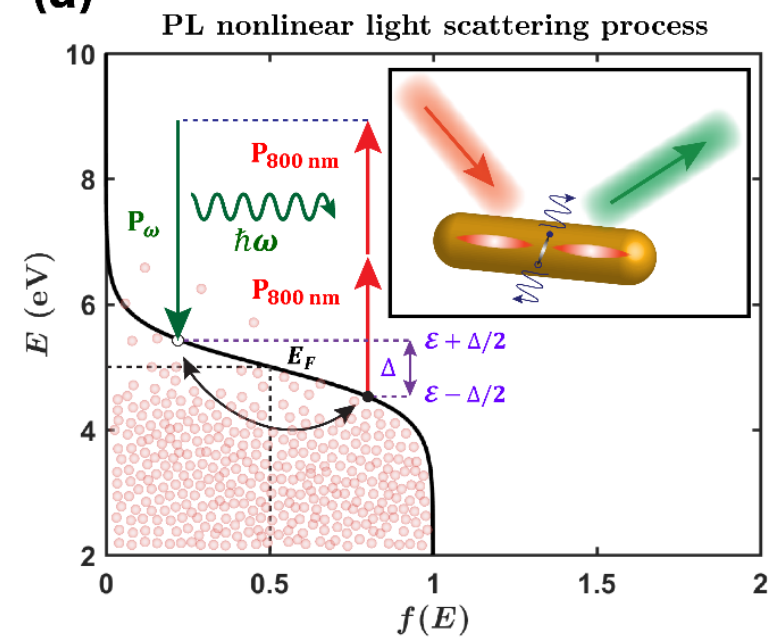

(b)

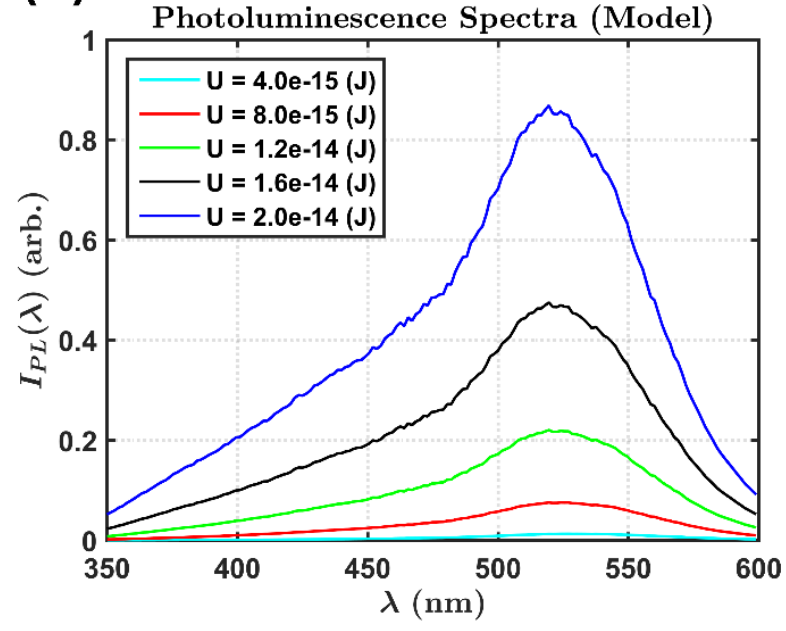

Figure 5. (a) Energy diagram of the PL nonlinear light scattering process and (inset) a physical illustration of the $2^{\text {nd }}$ order plasmon excitation and the PL generation. (b) Calculated scattered 
light spectrum using the values of $\gamma_{e e}$ and $U_{a b s}$ obtained from the SHG and THG experiments. The spectral line shape is determined by the (structure dependent) absorption spectrum of AuNRs, i.e., the measured UV-VIS spectrum.

\section{FUNDING SOURCES:}

This work was supported by the National Science Foundation through NSF DMR 1120923. The work at Brookhaven National Laboratory was supported by the Materials Science and Engineering Divisions, Office of Basic Energy Sciences of the U.S. Department of Energy under Contract No. DESC0012704. Electron holography experiments were carried out in part at the Center for Functional Nanomaterials, Brookhaven National Laboratory. 


\section{ABBREVIATIONS:}

SPR, surface plasmon resonances; PL, multi-photon luminescence; TPL, two-photon luminescence; THG, third-harmonic generation; SHG, second harmonic generation; AuNR, gold nanorod; CTAB, cetyltrimethylammonium bromide

\section{REFERENCES:}

(1) Mohamed, M. B.; Volkov, V.; Link, S.; El-Sayed, M. a.The `lightning’ Gold Nanorods: Fluorescence Enhancement of over a Million Compared to the Gold Metal. Chem. Phys. Lett. 2000, 317, 517-523.

(2) Kauranen, M.; Zayats, A.V.Nonlinear Plasmonics. Nat. Photonics 2012, 6, 737-748.

(3) Link, S.; Mohamed, M. B.; El-Sayed, M. A.Simulation of the Optical Absorption Spectra of Gold Nanorods as a Function of Their Aspect Ratio and the Effect of the Medium Dielectric Constant. J. Phys. Chem. B 1999, 103, 3073-3077.

(4) Jain, P. K.; Lee, K. S.; El-Sayed, I. H.; El-Sayed, M. a.Calculated Absorption and Scattering Properties of Gold Nanoparticles of Different Size, Shape, and Composition: Applications in Biological Imaging and Biomedicine. J. Phys. Chem. B 2006, 110, 72387248.

(5) Ni, W.; Kou, X.; Yang, Z.; Wang, J.Tailoring Longitudinal Surface Plasmon Wavelengths, Scattering and Absorption Cross Sections of Gold Nanorods. ACS Nano 2008, 2, 677-686.

(6) Fava, D.; Nie, Z.; Winnik, M. A.; Kumacheva, E.Evolution of Self-Assembled Structures 
of Polymer-Terminated Gold Nanorods in Selective Solvents. Adv. Mater. 2008, 20, 4318-4322.

(7) York, J.; Spetzler, D.; Xiong, F.; Frasch, W. D.Single-Molecule Detection of DNA via Sequence-Specific Links between F1-ATPase Motors and Gold Nanorod Sensors. Lab Chip 2008, 8, 415-419.

(8) Wang, H.; Huff, T. B.; Zweifel, D. a; He, W.; Low, P. S.; Wei, A.; Cheng, J.-X.In Vitro and in Vivo Two-Photon Luminescence Imaging of Single Gold Nanorods. Proc. Natl. Acad. Sci. 2005, 102, 15752-15756.

(9) Huang, X.; El-Sayed, I. H.; Qian, W.; El-Sayed, M. A.Cancer Cell Imaging and Photothermal Therapy in the near-Infrared Region by Using Gold Nanorods. J. Am. Chem. Soc. 2006, 128, 2115-2120.

(10) Durr, N.; Larson, T.; Smith, D.; Korgel, B.; Sokolov, K.; Ben-Yakar, A.Two-Photon Luminescence Imaging of Cancer Cells Using Molecularly Targeted Gold Nanorods. Nano Lett. 2007, 7, 941-945.

(11) Boyd, G. T.; Yu, Z. H.; Shen, Y. R.Photoinduced Luminescence from the Noble Metals and Its Enhancement on Roughened Surfaces. Phys. Rev. B 1986, 33, 7923-7936.

(12) Farrer, R. a.; Butterfield, F. L.; Chen, V. W.; Fourkas, J. T.Highly Efficient MultiphotonAbrortion-Induced Luminescence from Gold Nanoparticles. Nano Lett. 2005, 5, 11391142.

(13) Tong, L.; Wei, Q.; Wei, A.; Cheng, J.-X.Gold Nanorods as Contrast Agents for Biological 
Imaging: Optical Properties, Surface Conjugation and Photothermal Effects. Photochem. Photobiol. 2009, 85, 21-32.

(14) Lippitz, M.; Dijk, M. a; Orrit, M.Third-Harmonic Generation from Single Gold Nanoparticles. Nano Lett. 2005, 5, 799-802.

(15) Schwartz, O.; Oron, D.Background-Free Third Harmonic Imaging of Gold Nanorods. Nano Lett. 2009, 9, 4093-4097.

(16) Dadap, J. I.; Shan, J.; Eisenthal, K. B.; Heinz, T. F.Second-Harmonic Rayleigh Scattering from a Sphere of Centrosymmetric Material. Phys. Rev. Lett. 1999, 83, 4045-4048.

(17) Dadap, J. I.; Shan, J.; Heinz, T. F.Theory of Optical Second-Harmonic Generation from a Sphere of Centrosymmetric Material: Small-Particle Limit. J. Opt. Soc. Am. B 2004, 21, 1328-1347.

(18) Hao, E. C.; Schatz, G. C.; Johnson, R. C.; Hupp, J. T.Hyper-Rayleigh Scattering from Silver Nanoparticles. J. Chem. Phys. 2002, 117, 5963-5966.

(19) Russier-Antoine, I.; Benichou, E.; Bachelier, G.; Jonin, C.; Brevet, P. F.Multipolar Contributions of the Second Harmonic Generation from Silver and Gold Nanoparticles. $J$. Phys. Chem. C 2007, 111, 9044-9048.

(20) Bachelier, G.; Russier-Antoine, I.; Benichou, E.; Jonin, C.; Brevet, P.-F.Multipolar Second-Harmonic Generation in Noble Metal Nanoparticles. J. Opt. Soc. Am. B 2008, 25, 955.

(21) Butet, J.; Duboisset, J.; Bachelier, G.; Russier-Antoine, I.; Benichou, E.; Jonin, C.; Brevet, 
P.-F.Optical Second Harmonic Generation of Single Metallic Nanoparticles Embedded in a Homogeneous Medium. Nano Lett. 2010, 10, 1717-1721.

(22) Hubert, C.; Billot, L.; Adam, P.-M.; Bachelot, R.; Royer, P.; Grand, J.; Gindre, D.; Dorkenoo, K. D.; Fort, A.Role of Surface Plasmon in Second Harmonic Generation from Gold Nanorods. Appl. Phys. Lett. 2007, 90, 181105.

(23) Beversluis, M.; Bouhelier, A.; Novotny, L.Continuum Generation from Single Gold Nanostructures through near-Field Mediated Intraband Transitions. Phys. Rev. B 2003, 68, $1-10$.

(24) Imura, K.; Nagahara, T.; Okamoto, H.Near-Field Two-Photon-Induced Photoluminescence from Single Gold Nanorods and Imaging of Plasmon Modes. J. Phys. Chem. B 2005, 109, 13214-13220.

(25) Bouhelier, A.; Bachelot, R.; Lerondel, G.; Kostcheev, S.; Royer, P.; Wiederrecht, G. P.Surface Plasmon Characteristics of Tunable Photoluminescence in Single Gold Nanorods. Phys. Rev. Lett. 2005, 95.

(26) Knittel, V.; Fischer, M. P.; DeRoo, T.; Mecking, S.; Leitenstorfer, A.; Brida, D.Nonlinear Photoluminescence Spectrum of Single Gold Nanostructures. ACS Nano 2015, 9, 894900.

(27) Panasyuk, G. Y.; Schotland, J. C.; Markel, V. A.Classical Theory of Optical Nonlinearity in Conducting Nanoparticles. Phys. Rev. Lett. 2008, 100.

(28) Nik, B.; El-Sayed, M.Preparation and Growth Mechanism of Gold Nanorods (NRs) Using 
Seed - Mediated Growth Method. Chem. Mater. 2003, 15, 1957-1962.

(29) Kim, J.-Y.; Lien, M.-B.; Han, M.-G.; Zhu, Y.; Norris, T.; Kotov, N.Charge Anisotropy of Gold Nanorods. In 251st American Chemical Society National meeting \& Exposition; American Chemical Society: San Diego, 2016; p. 244.

(30) Völkl, E.; Allard, L. F.; Joy, D. C.Introduction to Electron Holography; Springer US, 1999; Vol. 53.

(31) Kim, J.-Y.; Lien, M.-B.; Han, M.-G.; Magonov, S.; Zhu, Y.; Ferguson, H.; Norris, T.; Kotov, N.Hidden Asymmetry of Gold Nanorods. (submission to Nat. Nanotechnology) 2017.

(32) Boyd, R. W.Nonlinear Optics; 2008.

(33) Groeneveld, R. H. M.; Sprik, R.; Lagendijk, A.Femtosecond Spectroscopy of ElectronElectron and Electron-Phonon Energy Relaxation in Ag and Au. Phys. Rev. B 1995, 51, 11433-11445.

(34) Perner, M.; Bost, P.; Lemmer, U.; vonPlessen, G.; Feldmann, J.; Becker, U.; Mennig, M.; Schmitt, M.; Schmidt, H.Optically Induced Damping of the Surface Plasmon Resonance in Gold Colloids. Phys. Rev. Lett. 1997, 78, 2192-2195.

(35) Lin, Z.; Zhigilei, L.V.; Celli, V.Electron-Phonon Coupling and Electron Heat Capacity of Metals under Conditions of Strong Electron-Phonon Nonequilibrium. Phys. Rev. B 2008, 77.

(36) Verellen, N.; Denkova, D.; Clercq, B.De; Silhanek, A.V.; Ameloot, M.; Dorpe, P.Van; 
Moshchalkov, V.V.Two-Photon Luminescence of Gold Nanorods Mediated by Higher Order Plasmon Modes. ACS Photonics 2015, 2, 410-416.

(37) Agarwal, G. S.Quantum Electrodynamics in the Presence of Dielectrics and Conductors. I. Electromagnetic-Field Response Functions and Black-Body Fluctuations in Finite Geometries. Phys. Rev. A 1975, 11, 230-242.

(38) Agarwal, G. S.Brownian Motion of a Quantum Oscillator. Phys. Rev. A 1971, 4, 739-747.

(39) Link, S.; Ei-Sayed, M. A.Spectroscopic Determination of the Melting Energy of a Gold Nanorod. J. Chem. Phys. 2001, 114, 2362-2368.

\section{GRAPHICAL TABLE OF CONTENTS:}

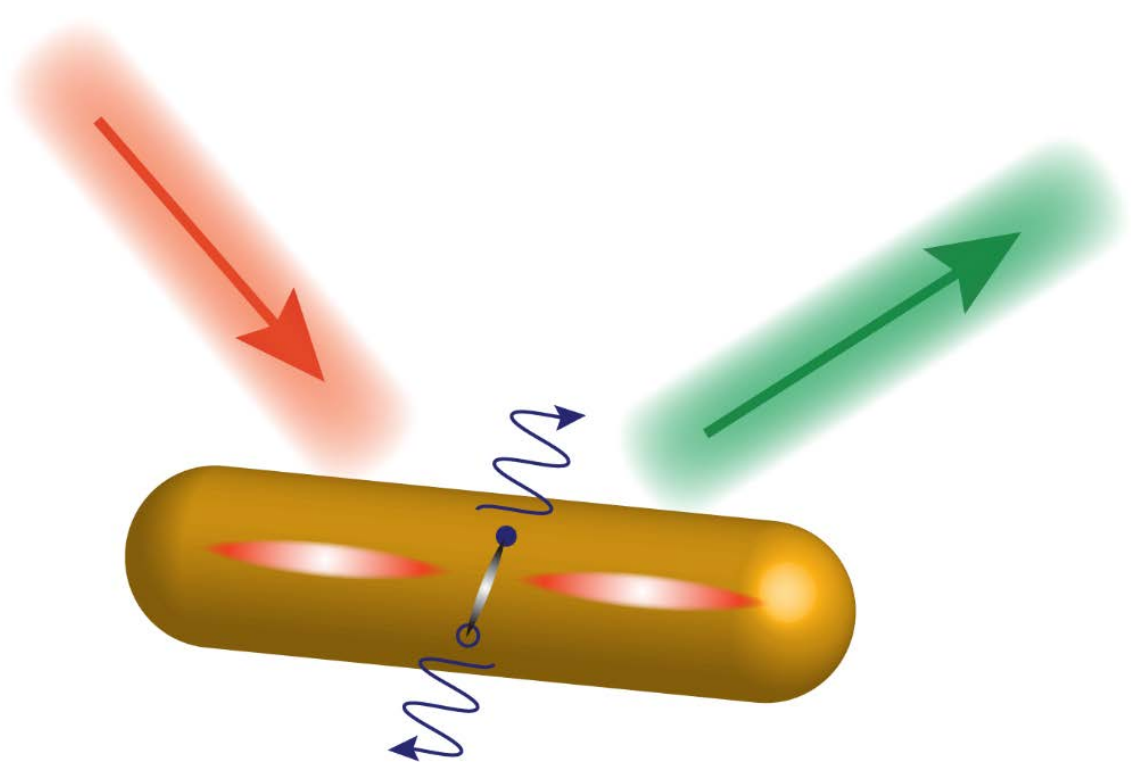

